\title{
Embedded system design for a multi variable input operations
}

\author{
Niranjan N. Parandkar,
}

\begin{abstract}
There are several multivariable input systems with one or more outputs exist in nature or designed and implemented successfully artificially. The input parameters of the system may or may not bear any relationship with each other however, output or outputs of the system definitely possess fixed relationship with input parameters. For every engineering student, understanding above concept is very important. An attempt has been made to develop a tool which will improve the understanding of such concept named as $e$ Stacapult by the author based on the principle of Catapult and similar type of tool developed as Statapult. The data is collected by performing some experiments and collected data is analyzed using softwares like $M A T L A B$ or DOE Wisdom. The tool can have wide applications in the field of training, Quality management, Six Sigma etc.
\end{abstract}

Index Terms: - Statapult, e-Stacapult, Multi variable system, Data collection and data analysis, Quality management.

\section{INTRODUCTION}

An observer who minutely observers the surrounding can lead to the conclusion that nature itself is having infinite number of systems consisting of number of inputs and one or more outputs. The input parameters of the system may be or may not be related to each other. But system may have output or outputs which definitely bears a particular relationship with variable input parameters.

Consider a case of crop growth in agriculture sector. The rate of growth of crop depends on various parameters like quality of soil, quality of seed quality and quantity of water, relative humidity, surrounding temperature and its variation with respect to time etc. Out of these, parameters like quality of seed and quality of soil are having fixed value and other are variable parameters. Variations like water quality can be adjusted manually, but effect of humidity and temperature occur naturally (However, in case of green house, they are adjusted manually).

Infinite number of systems have been designed and implemented in industrial, domestic and commercial environment. e.g. in case of washing machine, quality of washing which is output of the system depends on its input parameters like amount and quality of detergent, temperature of water, Number of washing and drying cycles, time for each cycle etc. These parameters are set by operator himself and inputoutput relationship is as per the designed program prepared by design engineer.

One of the earlier systems designed historically is the invention of Catapult. The catapult was invented by the famous physicist and mathematician Archimedes, which was developed to throw heavy stones on enemy ships for their demolition. In this case, the throw of stone was proportional to weight of stone, angle of throw etc.

For an engineering student, learning multi variable control system, the understanding of system operation is difficult. By merely specifying the input parameters by letters like $\alpha 1, \alpha 2, \alpha 3, \beta 1, \beta 2, \beta 3$ etc, the system visualization is difficult. Understanding will be better if the student handles a sample system designed for few input variable and one of the outputs which is measurable. NCMR LLC, which is a company from United States of America, had developed a tool called Statapult. However Statapult is a fully manual system and the author had provided some modifications so that some operations are performed automatically. The author had named it as e- Stacapult, since it has taken aid of electronic system electronically. The data collected is processed using MATLAB or DOE Wisdom software to achieve desired results.

The quality controlling process is also a system with multi variable input for a product on which the quality of final product depends. So the tool is also useful for training in quality controlling techniques like Six Sigma etc.

\section{Statapult System}

Using basic principles of Catapults, Statapult system is developed. It consists of two supporting members, between which a support arm is fixed. A movable arm is hinged at the rear end of support member, capable of moving at angular distance and the angle of movement can be adjusted as per the scale fixed on support members. This arm rests on a stopper and it is possible to set stopper position as ST1, ST2, ST3, ST4. 
The fixed arm carries four support positions S1, S2, S3, S4 which can be adjusted by using a peg. Similarly to adjust stopper position, another peg is provided. The movable arm carries 4 hooks which act as tension positions T1, T2, T3, T4 on which a rubber band slides. The same arm also carries a cup at its top, in which balls with different weights can be placed. An elastic rubber band is located between a fixed hook, support pin, and tension pin.

When the ball is kept in the cup, and the arm is pulled back at desired angle with stopper pin located at desired location. When the arm is released, it strikes the stopper pin due to the tension force generated by stretching the elastic band. Due to striking, the ball is thrown and the bouncing position of the ball depends on the inclination angle, the position of stopper pin and the tension in the rubber band. The tension in the rubber band depends on the stretching of rubber band and ultimately on support position and tension position.

In this system, the support positions, stopper positions, tension positions and angle of inclination are input variables and the first bounce distance is the output of the system. So there can be various input value sets for same output value. An effort has been made to add some degree of automation in the system and the automated system is named as "e-Stacapult".

\section{E-STACAPULT}

Figure 1 shows an e-Stacapult system which is modification of Statapult system. The following modifications have been designed and implemented.

1. Provision of a stepper motor to pull the tension arm at desired position.

2. Provision of an electromagnet to lock the arm at desired angle position and release it by deactivating electromagnet.

3. Provision of hand held LCD display and keypad unit to enter the input and output parameters and transferring data to the computer using a serial cable.

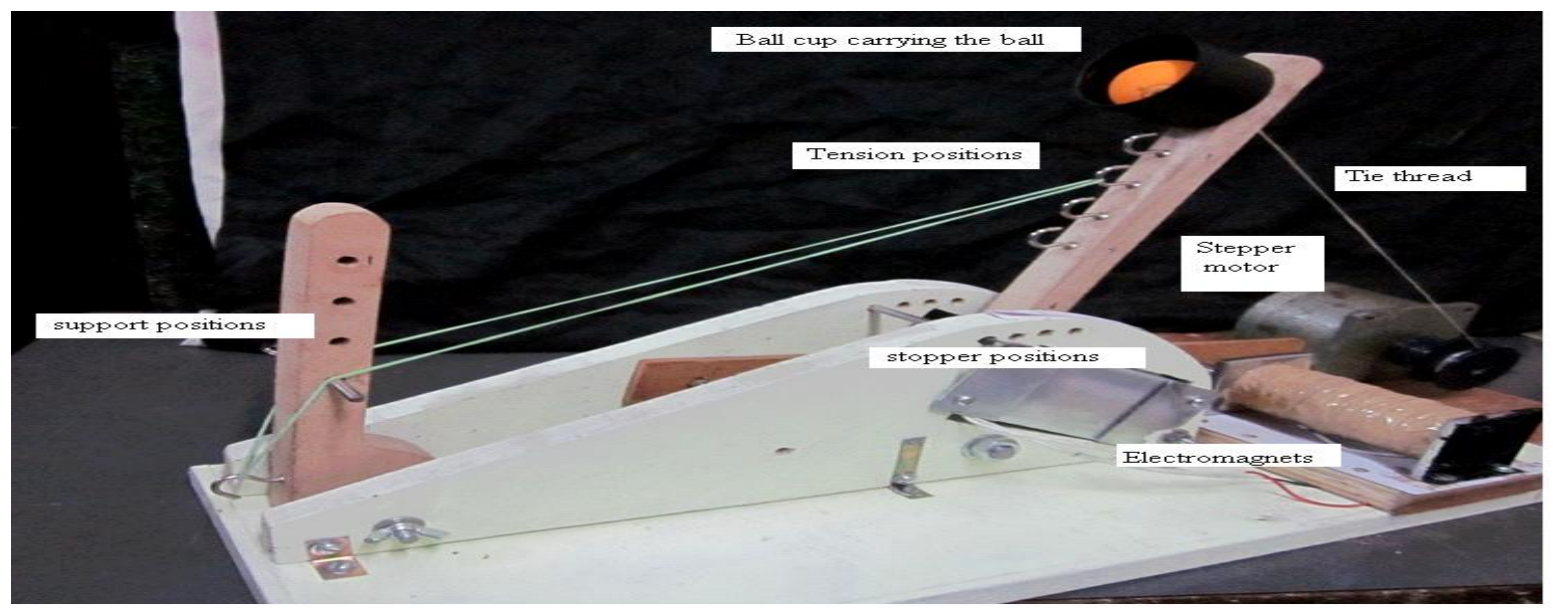

Figure 1.e-Stacapult system

All these provisions have been suggested to reduce any error due to human operations and make the system user friendly. ARM processor LPC2148 is used to facilitate above operations.

The data collected can be processed using MATLAB or DOE Wisdom software. It is possible to devise sets of components which can give same output.

\section{A.System Architecture}

The section discusses about different building blocks of e-Stacapult system. System includes various engineering aspects like mechanical design, electrical- electronic circuitry, stepper motor assembly, display module, transmission module. Mechanical assembly is already explained in previous section. Figure 2 shows electronic components and their role in this system.

\section{B. ARM Processor}

An automation in e-Stacapult is achieved by using micro-controller LPC2148. It belongs to ARM 7 family. ARM stands for Advanced RISC Machines. It is 32 bit processor with 32 bit ALU and a file of 32 bit general purpose registers. It can address 4 GB of memory. It has variety of addressing modes. It has three stage pipeline to execute instructions. Three stages are Fetch, Decode, Execute. ARM family of processors are 
mainly of low cost, low power applications like portable computer, cellular phones, automatic engines, embedded applications etc.

\section{Stepper Motor Control}

Stepper motor used to pull the tension arm, for which a tie

thread is connected to motor shaft and tension arm. Stepper

motor with step angle 1.8 degrees is used. Micro-controller is programmed to calculate number of steps required to reach desired angle positions. The stepper motor controller IC is used to meet the current requirement of motor.

\section{Electromechanical relay}

An electromechanical relay is used as switch to turn ON and OFF electromagnet. When tension arm reaches required

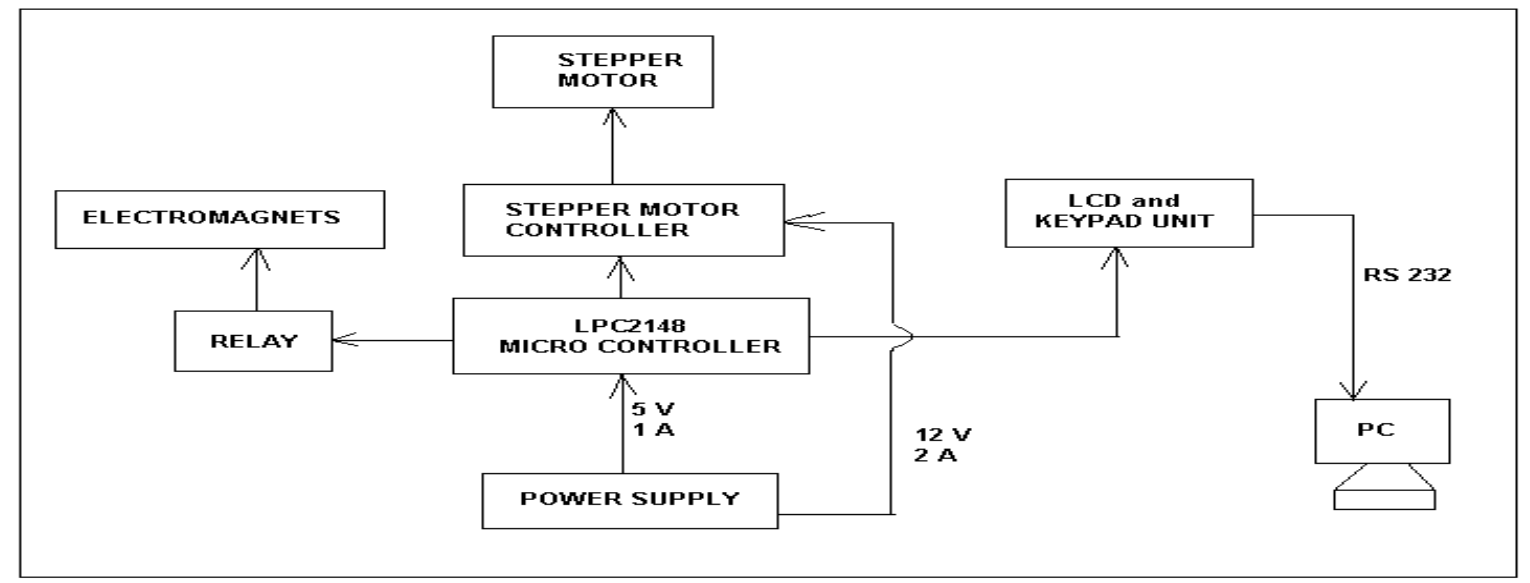

Figure 2. System architecture of e-Stacapult

angle position, electromagnet turns $\mathrm{ON}$ and holds the tension arm. After some predefined time the supply is removed OFF and electromagnet turns OFF, which effectively strikes tension arm to striker position. This switching is achieved using electronic relay.

\section{E. Display module and Data Transmission}

LCD and keypad module are interfaced so that operator enters input and output parameters. RS 232 serial transmission cable is used for transmitting data to computer using serial communication.

\section{OPERATING SEQUENCE}

The section focuses on how the system is going to work. A stepwise flow of all the operations is mentioned below.

1. When power supply is turned ON, operator is asked to enter the input parameters. He should enter the angle positions ranging from 100 degrees to 160 degrees with the difference of 10 degrees. Also Support, Tension, Stopper positions are to be entered between 1 to 4 .

2. Depending on the value of angle, micro-controller will calculate number of steps required by the stepper motor to be covered to reach required angle position.

3. Motor starts rotating and effectively tie thread that joins motor shaft and tension arm pulls tension arm up to angle position.

4. After reaching to that position, an electronic relay is turned ON, which switches ON electromagnets. These electromagnets hold tension arm for predefined time. Meanwhile stepper motor rotates in opposite direction unwinding the tie thread.

5. Once predefined time is elapsed, relay supply is removed OFF and thus electromagnets are also turned OFF. 6. Now there is no effective force acting to hold the lever arm. Thus due to tension exerted by rubber band, tension arm 
6.

str strikes the stopper position because of which the ball kept in the ball cup is thrown away.

Str 7. Operator measures the distance of the throw and enters the distance along with input parameters set using keypad and LCD.

8. Using serial transmission, all the data is transmitted to the computer connected by RS 232 cable.

9. Further Data Processing is done on that computer.

\section{DATA COLLECTION}

A sample sets of data collected is shown in figure 4. Each set includes any of the positions of support, tension, stopper positions and any value of angle positions in degrees. It should be noted that, there could be large number of such sets. Even for same output i.e. distance, more than one sets of different parameters are found.

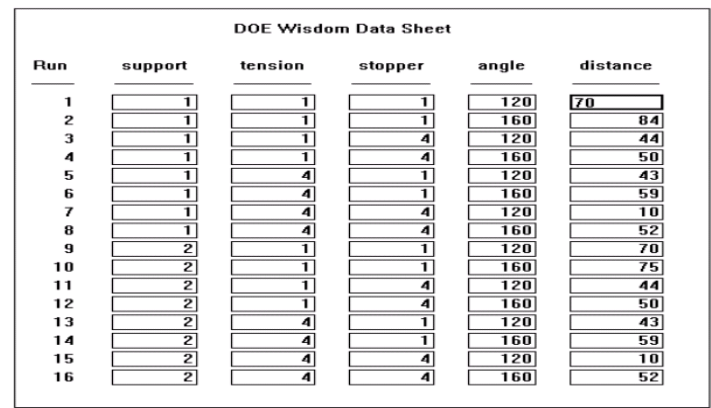

Figure 3. Data collection

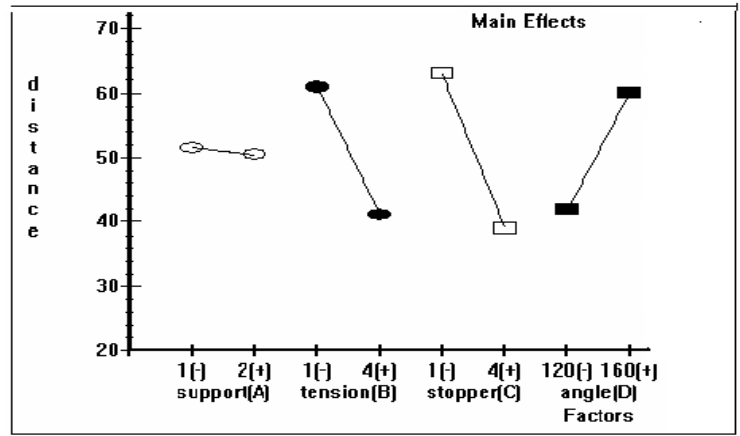

Figure 4. Main effects graph

DOE Wisdom software is used where data is collected and analyzed.

VI. DATA ANALYSIS

The section deals with a statistical study of data collected, from all the sets of input and output readings, various graphs are plotted. The graphs like Main Effect Graph, response surface etc. help us to study the effects of each input parameters on output. Figure 4 and figure 5 show these graphs.

The graph above explains out of four variable parameters which one affects output maximum or least, depending on the slope drawn on $\mathrm{X}$ axis within its upper and lower limits. Though wide range in the distance is observed, it is observed that variation in tension, stopper and angle values affect much more than that of support position value.

Similarly, response surface shown below, plots the generalized behavior of all input parameters and corresponding output values in a single surface plane.

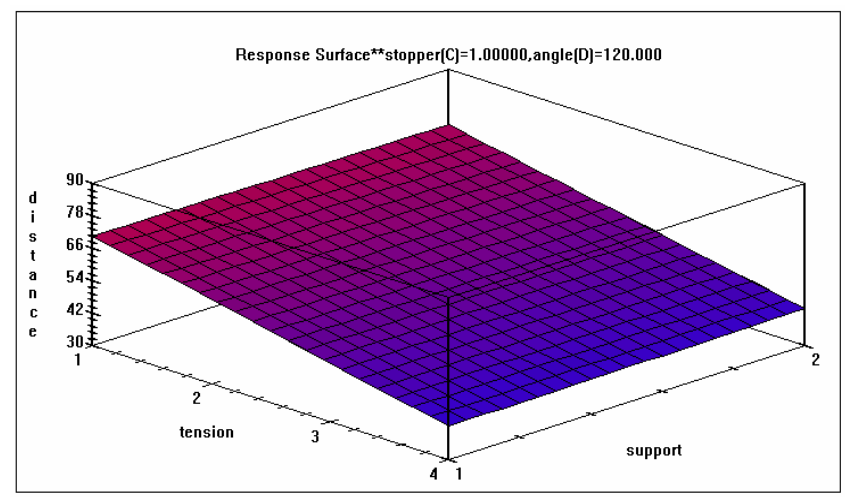

Figure 5. Response Sruface graph 


\section{A. Prediction of input values for desired output}

It is explained earlier that DOE Wisdom software is used to analyze the effects of multiple inputs on the output. Up to this stage, database of almost all permutation and combinations of four inputs and respective output is fed to the software. It is already explained in previous section effects of inputs on output.

But it is possible to predict the values of support, tension, stopper positions and angle value to meet the desired distance. Consider following figure 6 .

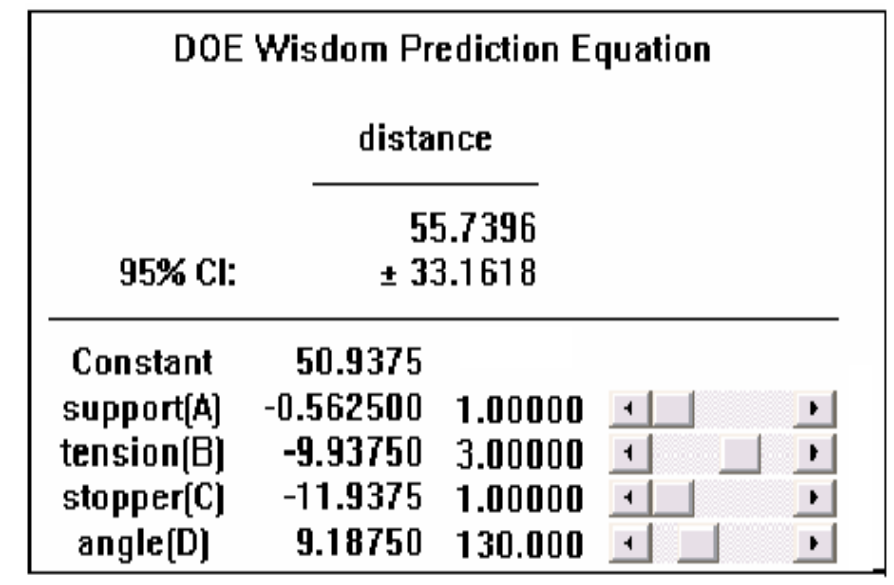

Figure 6. Prediction of parameters

For distance to be covered by a ball of say $55 \mathrm{~cm}$, it is intended to find the values of support,tension, stopper and angle values. Using previous observations, the values of these parameters are calculated. Support position 1,Tension position 3, Stopper position 1 and angle of 130 degrees are the values of inputs which lead to desired distance $55 \mathrm{~cm}$.

Also using reverse engineering, these results are verified on actual kit of e- Stacapult.

\section{CONCLUSION}

The e- Stacapult system is designed, studied, and implemented successfully. As mentioned, it helps to understand the effects of input parameters on the output. Also, concept of multi variable input system is understood.

Data analysis is done using DOE Wisdom software. Using the analyzed data, it can be calculated that for required desired output how the parameters are to be set. The simulated results are verified on actual eStacapult system.

[1] www.ncmrcompany.com

\section{References}

[2] www.sixsigmaproductsgroup.com

[3] Math and measurement- The Statapult lab journal

[4] Statapult Exercise, Six Sigma Green Belt Certification Course

[5] Catalogue of Statapult

[6] "Electric Machines And Power Systems" by Vincent Del Toro, PHI Publications. 